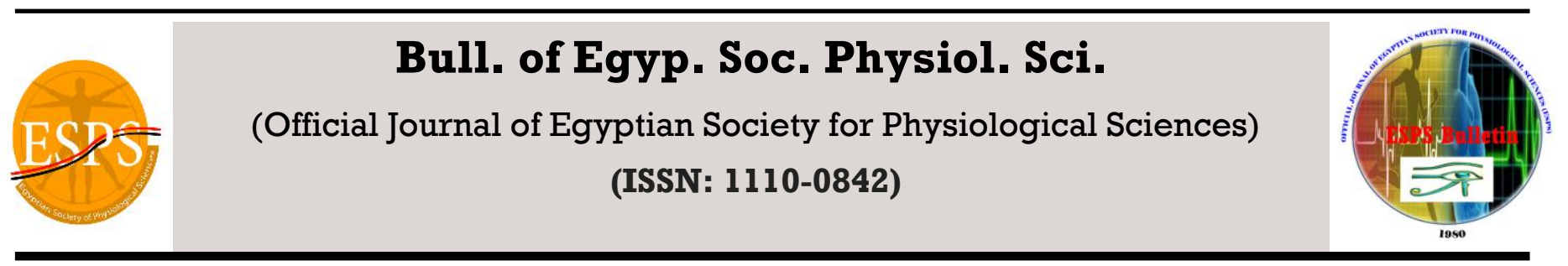

\title{
Closed and Open Loop Mechanisms Controlling Arterial Blood Pressure in Waking State in Human and Experimental Animal
}

\author{
David C. Randall ${ }^{1}$ and David R. Brown ${ }^{1}$ \\ ${ }^{1}$ Department of Physiology, College of Medicine, University of Kentucky, Lexington, KY
}

Received: March 10, 2014

Accepted: April 1, 2014

Available online: April 7, 2014

\section{Keywords}

Open and closed loop

Mechanisms

ABP

Waking

Man

Experimental animals

\section{Abstract}

Time-honored indices of classical, closed loop arterial blood pressure $\left(\mathrm{BP}_{\mathrm{a}}\right)$ control have recently been supplemented by analyses thought to be sensitive to both closed and open loop mechanisms. These newer tools include, among others, power spectral analyses of heart rate (HR) and $\mathrm{BP}_{\mathrm{a}}$, and the cross-correlation between changes in $\mathrm{BP}_{\mathrm{a}}$ and changes in $\mathrm{HR}$ or $\mathrm{R}-\mathrm{R}$ interval. The 'baroreceptor effectiveness index' (BEI) is significantly larger in supine, healthy individuals as compared to diabetic patients with peripheral neuropathy. Such findings give promise that BEI may provide a means to assess the development of autonomic neuropathy in diabetic or in other at-risk patients. The cross-correlation between $\mathrm{BP}_{\mathrm{a}}$ and heart rate in man includes a peak where the two are positively correlated with HR changes leading pressure changes, suggestive of open loop control, and a sharp nadir where the two are negatively correlated and $\mathrm{BP}_{\mathrm{a}}$ changes precede HR changes, suggestive of closed loop control. In rat the positive cross-correlation depends upon intact spinal sympathetic pathways. The magnitudes of both the positive and negative cross correlations are weaker in tetraplegic patients as compared to neutrally intact subjects. Both peaks remain relatively strong in healthy subjects during the challenge of head up tilt, but decline precipitously in tetraplegic patients. The recognition that both closed and open loop mechanisms are operative and functionally important suggests multiple additional possible etiologies for cardiovascular dysfunctions, including hypertension. The advent of non-invasive means to quantify autonomic function promises new insights into control of the cardiovascular system in health and disease.

Corresponding author: David C. Randall, Department of Physiology, College of Medicine, University of Kentucky, Lexington, KY.

Email address: randall@email.uky.edu; Fax: 859-323-1070 
In their classic study Smyth et al. ${ }^{1}$ inaugurated a new approach to the examination of the nervous control of arterial blood pressure $\left(\mathrm{BP}_{\mathrm{a}}\right)$ in the human subject, an area of research that continues unabated after almost half a century. They quantified the action, or 'sensitivity', of the baroreflex in the sleeping vs. the awake state using the slope of a best-fit linear line produced by plotting successive values of systolic blood pressure (SBP) against pulse interval for the next beat during acute, pharmacologically-induced pressor events. More specifically, $\mathrm{BP}_{\mathrm{a}}$ recordings from a catheter in the brachial artery were made during moderate $(25-35 \mathrm{~mm} \mathrm{Hg})$ increases in pressure induced by intravenous injections of angiotensin. The cardiac slowing, in msec., per $\mathrm{mm} \mathrm{Hg}$ rise in systolic pressure in 5 normotensive subjects increased from $8.4 \pm 6.0$ (mean $\pm \mathrm{SD}$ ) in the awake condition to $14.4 \pm 9.8 \mathrm{msec} / \mathrm{mm} \mathrm{Hg}$ while sleeping (computation by present author from data in Smyth et al.'s ${ }^{1}$ Table 2). The authors concluded that "...the baroreceptor reflex arc can be rapidly reset, particularly during sleep" (quoted from abstract).

The work by Smyth, et al. ${ }^{1}$ was invasive, requiring introduction of two 'lines' into the vasculature and administration of an exogenous drug to 'perturb' the baroreflex. Eckberg and Eckberg ${ }^{2}$ ingeniously used neck suction in young volunteers to perturb the baroreflex non-invasively and non-pharmacologically; the suction created "gradual progressive elevations in arterial pressure" (quoted from p. H638). They reported the reflex changes in P-P interval (i.e., interval between Pwaves, analogous to R-R interval) associated with these pressure ramps and concluded that "...the pattern of sinus node responses during transitions of arterial pressure results from a complex interplay among several factors, including baroreflex responsiveness, base-line heart rate, the rate of decay of baroreflex inhibition, and the biphasic nature of sinus node responses to inhibitory cholinergic interventions" (quoted from abstract).

Both the 'classic' pharmacologic and the neck-suction perturbations require fairly substantive restrictions in a subject's circumstances. The more recent 'spontaneous sequence technique' (e.g., 3-5) assesses $\mathrm{BP}_{\mathrm{a}}$ regulation by computerscanning on-going recordings of arterial pressure (usually beat-by-beat SBP, often obtained by a non-invasive technique such as pulse tonometry (e.g., via the Finapres)) for a series of successive three or four heart beats where pressure spontaneously increases (or decreases) and R-R interval (RRI) lengthens (or shortens); a one (or more) beat delay is often imposed between the pressure change and the interval measurement (see 5 or 6 for a review). 
Restrictions are often imposed upon the characteristics of a recording before accepting a given series of beats as a 'baroreflex sequence' such as in our recent study $^{7}$ where we identified sequences of three or more consecutive heartbeats in which progressively increasing (or decreasing) SBP with at least $1 \mathrm{~mm} \mathrm{Hg}$ beatto-beat change was followed within one heartbeat by progressively lengthening (or shortening) of RRI with at least 4-ms beatto-beat change. We accepted a series of beats as a baroreflex sequence if the correlation coefficient of the regression line between SBP and RR interval within the sequence was 0.85 or greater. As in the pharmacologic approach, this sequence technique accepts the slope of the regression line between the SBP and RRI values as an index of the sensitivity, or gain, of the baroreflex.

Such sequences could, of course, occur purely by chance, rather than as a mechanistic consequence of the action of the baroreflex. Note, for example, if the second beat is an increase (or decrease) relative to the first, then there is a $50 \%$ chance that the third beat will also be an increase (or decrease) so, neglecting any restrictions that may be placed on a sequence (e.g., change in SBP must exceed $1 \mathrm{~mm} \mathrm{Hg}$ ), there is a relatively high probability of finding a sequence of 3 progressively increasing (or decreasing) SBP pressure ramps. Blaber, et al. ${ }^{8}$ constructed 'surrogate data sets' to test whether the occurrence of 'baroreflex sequences' actually reflected simply random events. These sets were created from original recordings in healthy men and women in which the power spectrum of the original set was preserved but the phase relationship (i.e., between SBP and RRI) was randomized in the scrambled set (which they called the 'isospectral' set), or in a second surrogate set in which each of the original data points was shuffled randomly (i.e., the 'isodistribution surrogate'). They reported $^{8}$ that " $(t)$ here was a significantly greater total number of spontaneous baroreflex sequences in the original data then either the isospectral or the isodistribution surrogates..." (quoted from p. H1685). That is, they argue that their findings provided strong evidence that spontaneous baroreflex sequences were physiological rather than merely chance events.

In actual fact, SBP / RRI baroreflex sequences, which reportedly occur at about $80 /$ hour in $\operatorname{man}^{9}$, are often intermixed with progressive SBP changes that are not associated with the expected RRI changes (e.g., 9). In light of this Di Rienzo, et al. ${ }^{9}$ offered a new index, the 'baroreflex effectiveness index' (BEI) which they defined as the ratio between the number of SBP ramps followed by the respective reflex RRI ramps and the total number of SBP 
ramps (i.e., irrespective of the nature of any time-coupled changes in RRI) observed within a given time window. They reported ${ }^{9}$ from their study of 14 healthy subjects that the BEI was 0.21 (i.e., 21\%) with marked day-night variation ( 0.25 day, $\sim 0.15$ night). Moreover, the number of such ramps clearly depends upon an individual's HR: a higher rate would tend to increase the number of baroreflex sequences. In the Wang, et al. study $^{7}$ the number of SBP ramps normalized against on-going HR in supine, healthy subjects $(\mathrm{n}=11)$ was $0.13 \pm 0.02$ (/beat); the normalized number of baroreflex sequences in this group was $0.03 \pm 0.01$ while the BEI was $26 \pm 4.93(\%)$, similar to that reported by di Rienzo, et al ${ }^{9}$. The corresponding data for diabetic individuals with $(\mathrm{n}=8)$ distal symmetrical polyneuropathy (7) were $0.20 \pm$ 0.01 (/ beat), $0.02 \pm 0.01$ (/beat) and $9 \pm 5.1$ $(\%)$, respectively, indicating that in the presence of autonomic neuropathy there was a demonstrable decrease in baroreflex 'effectiveness'.

One additional time-domain technique is useful in analyzing the regulation of $\mathrm{BP}_{\mathrm{a}}$ in both awake rat and in man: the cross correlation between mean $\mathrm{BP}_{\mathrm{a}}\left(\mathrm{mBP}_{\mathrm{a}}\right)$ and $\mathrm{HR}{ }^{10,11}$. This approach is advantageous in the sense that it assesses the relationship across an entire data set, not specifically within a given 3 or 4 beat sequence. In undisturbed rat the major peak in the relationship, perhaps surprisingly, is positive (i.e., denoting a direct relationship between $\mathrm{mBP}_{\mathrm{a}}$ and $\mathrm{HR}$ ) with $\mathrm{HR}$ changes, again perhaps unexpectedly, leading changes in $\mathrm{mBP}_{\mathrm{a}}{ }^{11}$. In rat there is also a nadir in the relationship where $\mathrm{mBP}_{\mathrm{a}}$ changes lead HR changes, but even here the cross correlation remains positive. It is only after complete transection of the spinal cord (in rat), interrupting the sympathetic pathways, but leaving vagal control of HR intact, that the 'expected' negative cross correlation appeared with $\mathrm{mBP}_{\mathrm{a}}$ changes leading $\mathrm{HR}$ changes ${ }^{11}$. The cross correlation in the human has two notable features ${ }^{10}$ : a positive correlation where, as in rat, HR changes lead pressure changes plus - unlike rat - a strong negative correlation with pressure changes leading HR changes; this latter feature presumably reflects 'classical' baroreflex function. The magnitude of the negative cross correlation increased during passive head up tilt (HUT) in neurally intact subjects, but decreased to near zero in tetraplegic patients ${ }^{10}$.

Frequency domain or power spectral approaches have also been utilized to assess arterial $\mathrm{BP}_{\mathrm{a}}$ control in both unanesthetized animal and awake human studies. Brown, et al. ${ }^{12}$ and Julien, et al. ${ }^{13}$ reported that in anaesthetized rats renal sympathetic nerve activity and arterial $\mathrm{BP}_{\mathrm{a}}$ were highly coherent at a frequency in the vicinity of 0.4 Hz. Moreover, this high correlation can be explained theoretically on the basis of a 
'resonance' in the baroreflex ${ }^{14,15}$ and, in fact, was significantly decreased by sinoaortic denervation ${ }^{3,13,16,17}$. The corresponding frequency in the human is $\sim 0.1 \mathrm{~Hz}$, and corresponds to the so-called Mayer Wave ${ }^{14}$.

The $\mathrm{BP}_{\mathrm{a}}$ power spectrum has other notable characteristics beyond the $0.4 \mathrm{~Hz}$ (rat) or $0.1 \mathrm{~Hz}$ (human) rhythm. In particular, plotting log of $\mathrm{mBP}_{\mathrm{a}}$ power vs. $\log$ of frequency based upon a Fourier analysis of multi-day pressure recordings via telemetry in awake rat $^{18}$ reveals a 'plateau' in the spectrum (i.e., essentially unchanged power across a range of frequency). This plateau extends from $\sim 2$ cycles / day (that is, starting just below the spectral peak resultant from the large light:dark or circadian variation) to ca. 1 cycle / hr. Power decreases linearly in two ranges across frequencies 'below' (i.e., lower frequency) the plateau, extending in the aggregate from 1 cycle / hr to about 60 cycles / hour. This log-log linear pattern is characteristic of ' $1 / \mathrm{f}$ ' behavior and can be quantified in terms of the slope, often abbreviated as ' $\beta$ '; this pattern is seen in systems that are 'self similar'.

Brown, et al. ${ }^{18}$ published a computer simulation that replicated a number of aspects of the actual arterial $\mathrm{mBP}_{\mathrm{a}}$ power spectrum for rat in the lower-frequency region (i.e., 2 cycles / day (or 0.083 cycles / hr) to $\sim 1$ cycle / 10 minutes). Their model simulated the mean arterial pressure time series as a random walk (i.e., such as Brownian motion, as though beat-by-beat $\mathrm{BP}_{\mathrm{a}}$ fluctuations conformed to a stochastic process); their model, however, constrained the permitted range of the random walk - as though the extent of the 'meandering' were curbed by the baroreflex - so that $\mathrm{mBP}_{\mathrm{a}}$ always remained within stated limits. In other words, the action of the baroreflex within the model did not 'allow' $\mathrm{mBP}_{\mathrm{a}}$ to 'wander' above or below the stated limits. In addition, for each 'heart beat' $\mathrm{mBP}_{\mathrm{a}}$ was constrained to change (i.e., $\Delta$ ) by a given number of ' $\mathrm{mm} \mathrm{Hg}$ '; this is similar to insisting that the immediate prior history of $\mathrm{mBP}_{\mathrm{a}}$ placed constraints on the current value. This means, in effect, that the cardiovascular system has a 'memory' imposed by its physical (i.e., hydraulic) characteristics. The resulting spectrum of the model's ' $\mathrm{mBP}_{\mathrm{a}}$ ' closely replicated the actual spectrum from rats (i.e., that were undisturbed in their home cages) when the allowed range was set to a physiologically realistic $85-115 \mathrm{~mm} \mathrm{Hg}$ and $\Delta$ was set to 2 'mm Hg', close to that value actually observed in the rat $(1.87 \pm 0.01 \mathrm{~mm} \mathrm{Hg}$, mean $\pm \mathrm{SD}$ ). The similarities included a plateau in the model where the slope $(0.207)$ closely mimicked the observed value $(0.32 \pm$ 0.28 between $0.083 / \mathrm{hr}$ to $1 / \mathrm{hr}$ ). What's more, the slopes of the ' $1 / \mathrm{f}$ ' regions of the spectrum (e.g., 1 to $6 / \mathrm{hr}$ ) generated by the 
model (1.707) mimicked the value determined in the freely ambulatory rat (1.80 \pm 0.16 ). Based on these findings the authors speculated that "...the baroreflex does not continuously and uncompromisingly dictate beat-by-beat pressure behavior but is aggressively called into play when pressure rises above or falls below allowed limits. That is, the empirical findings and theoretical model seem to be consistent with the possibility that those closed-loop systems impinging on arterial pressure, including, but by no means limited to, the baroreflex, are relatively disengaged from beat-by-beat pressure control so long as pressure 'wanders' within appropriate limits" (quoted from 18, p. H2823).

Seemingly in accord with the speculation offered by Brown, et al. ${ }^{18}$, results from assessments of $\mathrm{BP}_{\mathrm{a}}$ regulation using the spontaneous baroreflex sequence and/or BEI suggest, that, indeed, in the 'resting' human regulation of pressure by closed-loop mechanisms is relatively 'hands off' (i.e., the total number of baroreflex sequences is quite low). In fact, in presenting their baroreflex effectiveness index, Di Rienzo, et al. ${ }^{9}$ noted that "Application of the sequence technique over the years has shown that in healthy subject, not every SBP ramp is invariably followed by a reflex RRI ramp. This suggests that under physiological conditions, the baroreflex is not always effective in gaining control of the sinus node" (quoted from $\mathrm{p}$. 29), which observation then led them to propose the BEI. In fact, again as cited above from Wang, et al.'s findings ${ }^{7}$, the BEI is notably greater ( $26 \%$ in healthy subjects when supine) than the normalized sequence reports. Conversely BEI was notably lower in individuals with neuropathy as compared to healthy controls and failed to increase when the neuropathy subjects were challenged by $\mathrm{HUT}^{7}$.

Interestingly, while Brown, et al.'s ${ }^{18}$ stochastic model implies that the baroreflex may not consistently control HR, the findings of Burgess, et al. ${ }^{15}$, from the same laboratory, presented experimental and mathematical evidence that the baroreflex is active, at least within a limited bandpass. One possible explanation for this apparent inconsistency is that the $\mathrm{mBP}_{\mathrm{a}}$ rhythms resultant from the resonance continuously wax and wane as a subject's autonomic function adapts to on-going environmental and behavioral conditions. This is congruent with multiple reports that not all sequences are of barostatic origin. If so, then the occurrence of barostatic sequences may be dominated by the resonant $\mathrm{BP}_{\mathrm{a}}$ cycles. Certainly if the resonant cycles are present, then sequences of longer than one half of their cycle-length are not possible (e.g., $1 / 2 \mathrm{x}$ 2.5 sec. = maximum sequence duration is $1.25 \mathrm{sec}$. for $0.4 \mathrm{~Hz}$ rhythm in rat). 
Any further observations here must be made with the realization that it is impossible to untangle closed vs. open loop mechanisms in the intact organism. Nevertheless, if our speculation regarding the freedom of $\mathrm{BP}_{\mathrm{a}}$ to 'wander' is correct, the values for baroreflex gain as computed from the HR changes provoked by the classic pharmacologic tests would overestimate the closed loop control because the baroreflex would become 'engaged' by the sudden trending of $\mathrm{BP}_{\mathrm{a}}$ towards our supposed 'allowed limits'. Conversely, our speculations absolutely do not negate the possibility that other autonomic neural mechanisms participate aggressively in moment-by-moment $\mathrm{BP}_{\mathrm{a}}$ control. In particular, the immediate $\mathrm{BP}_{\mathrm{a}}$ response to a suddenly perceived behavioral challenge in rat derives directly from what we termed an 'open loop' mechanism (often called 'central command') that is able to respond more rapidly than classic closed-loop reflexes ${ }^{19}$. It seems very likely that those central nervous loci that co-ordinate the body's responses accompanying, for example, exercise would direct not only the muscular components of that activity, but would also concomitantly direct the respiratory, cardiovascular and other visceral aspects of the behavior. The cross correlation analysis cited previously could also be interpreted to support the supposed role of an open loop mechanism in on-going $\mathrm{BP}_{\mathrm{a}}$ control: in rat this mechanism is exercised primarily via sympathetic mechanisms and the nervous system 'falls back' on the remaining biofeedback pathway utilizing parasympathetic control of HR when the sympathetic pathways are dysfunctional. From this perspective, the human, where parasympathetic regulation of SA-nodal function is more robust than in rat, appears to depend in a more balanced fashion upon both closed and open loop regulatory mechanisms to optimize $\mathrm{BP}_{\mathrm{a}}$ for any given behavioral condition. Not surprisingly, it seems a great deal more remains to be learned about the on-going control of arterial pressure, including the possibility that conditions such as hypertension are, in fact, a 'learned behavior' (e.g., 20) via open loop controls.

\section{REFERENCES}

\section{Smyth HS, Sleight P, Pickering GW:}

Reflex regulation of arterial pressure during sleep in man. A quantitative method of assessing baroreflex sensitivity. Circ Res 24: 109-121, 1969.

2. Eckberg DL and Eckberg MJ: Human sinus node responses to repetitive, ramped carotid baroreceptor stimuli. Am J Physiol Regul Integr Comp Physiol 242: H638-H644, 1982. 
3. Bertinieri G, di Rienzo M, Cavallazzi A, Ferrari AU, Pedotti A, Mancia G: Evaluation of baroreceptor reflex by blood pressure monitoring in unanesthetized cats. Am J Physiol Heart Circ Physiol 254: H377-H383, 1988.

4. Di Rienzo M, Bertinieri G, Mancia G, Pedotti A: A new method for evaluating the baroreflex role by a joint pattern analysis of pulse interval and systolic blood pressure series. Med Biol Eng Comput 23 (suppl. I): 313-314, 1985.

5. Fritsch JM, Eckberg DL, Graves LD, Wallin BG. Arterial pressure ramps provoke linear increases of heart period in humans. Am J Physiol Regul Integr Comp Physiol 251: R1086-R1090, 1986.

6. Laude D, Elghozi J-L, Girard A, Bellard E, Bouhaddi M, Castiglioni P, Cerutti C, Cividjian A, Di Rienzo M, Fortrat J-O, Janssen B, Karemaker JM, Lefthériotis G, Parati G, Persson PB, Porta A, Quintin L, Regnard J, Rüdiger H, Stauss HM. Comparison of various techniques used to estimate spontaneous baroreflex sensitivity (the EuroBaVar study). Am J Physiol
Regul Integr Comp Physiol 286: R226R231, 2004.

7. Wang S, Randall DC, Knapp CF, Patwardhan AR, Nelson KR, Karounos DG, Evans JM. Blood pressure regulation in diabetic patients with and without peripheral neuropathy. Am J Physiol Regul Integr Comp Physiol 302: R541-R550, 2012.

8. Blaber AP, Yamamoto Y, Hughson RL. Methodology of spontaneous baroreflex relationship assessed by surrogate data analysis. Am J Physiol Heart Circ Physiol 268: H1682-H1687, 1995.

9. Di Rienzo M, Parati G, Castiglioni P, Tordi R, Mancia G, Pedotti A: Baroreflex effectiveness index: an additional measure of baroreflex control of heart rate in daily life. Am J Physiol Regul Integr Comp Physiol 280: R744-R751, 2001.

10. Aslan SC, Randall DC, Donohue KD, Knapp CF, Patwardhan AR, McDowell SM, Taylor RF, Evans JM. Blood pressure regulation in neurally intact human vs. acutely injured paraplegic and tetraplegic patients during passive tilt. Am. J. 
Physiol. Regul. Integr. Comp. Physiol., 292: R1146 - R1157, 2007.

11. Baldridge BR, Burgess DE, Zimmerman EE, Carroll JJ, Sprinkle AG, Speakman RO, Li S-G, Brown DR, Taylor RF, Dworkin S Randall DC Heart Rate-Arterial Blood Pressure Relationship in Conscious Rat before vs. after Spinal Cord Transection. Am. J. Physiol. Regul. Integr. Comp. Physiol. 283: R748-R756, 2002.

12. Brown DR, Brown LV, Patwardham A, Randall DC. Sympathetic activity and blood pressure are tightly coupled at $0.4 \mathrm{~Hz}$ in conscious rat. Am J Physiol Regul Integr Comp Physiol 267: R1378 - R1384, 1994.

\section{Julien C, Chapuis B, Cheng Y, Barres}

C: Dynamic interactions between arterial pressure and sympathetic nerve activity: role of arterial baroreceptors. Am J Physiol Regul Integr Comp Physiol 285: R834-R841, 2003.

\section{Bertram D, Barres C, Cuisinaud G,} Julien C: The arterial baroreceptor reflex of the rat exhibits positive feedback properties at the frequency of Mayer waves. J Phyiol 513: 251-261, 1998.
15. Burgess, DE, Hundley JC, Li S-G, Randall DC, Brown DR. A first-order differential-delay equation for the baroreflex predicts the $0.4 \mathrm{~Hz}$ blood pressure rhythm in rats. Am J Physiol Regul Integr Comp Physiol 273: R1878-1884, 1997.

\section{Barres C, Lewis SJ, Jacob HJ, Brody} MJ. Arterial pressure lability and renal sympathetic nerve activity are dissociated in SAD rats. Am J Physiol Regul Integr Comp Physiol 263:R639R646, 1992.

17. Julien C, Zhang Z-Q, Cerutti C, Barres C: Hemodynamic analysis of arterial pressure oscillations in conscious rats. J Autonomic Nerv Syst 50: 239-252, 1995.

18. Brown DR, Cassis LA, Silcox DL, Brown LV and Randall DC. Empirical and theoretical analysis of the extremely low frequency arterial blood pressure power spectrum in unanesthetized rat. Am. J. Physiol. Heart Circ. Physiol. 291: H2816 H2824, 2006.

\section{Randall, D.C., Brown DR, Brown LV,} Kilgore JM. Sympathetic nervous activity and arterial blood pressure 
control in conscious rat during rest and behavioral stress. Am J Physiol Regul Integr Comp Physiol 267: R1241 R1249, 1994.

\section{Harris AH, Gilliam WJ, Findley JD}

Brady JV. Instrumental conditioning of large-magnitude, daily, 12-hour blood pressure elevations in the baboon. Science 182: 175-177, 1973. 